\title{
Time-Of-Flight Mass Spectrometers Made in Switzerland: Examples of Mobile Applications
}

\author{
Manuel A. Hutterli, Veronika Pospisilova, and Marc Gonin
}

\begin{abstract}
Over the past decade, the technical requirements of analytical instrumentation have continuously risen driven by the demand for increasingly complex and demanding applications. TOFWERK, a Swiss company with their headquarters in Thun, has been at the forefront of this development by producing modular and ruggedized Time-Of-Flight Mass Spectrometers (TOFMS). They are often used to replace quadrupole mass analysers with more powerful TOF mass analysers. Starting with first customers in atmospheric sciences, TOFWERK TOFMS are now used across a wide range of research areas and lately also in industry. Here we present an overview of mobile applications in which TOFWERK TOFMS are in operation while being moved around in space.
\end{abstract}

Keywords: Chemical ionisation · Mobile applications · Real time measurement · Time-of-flight mass spectrometry $\cdot$ Volatile organic compounds

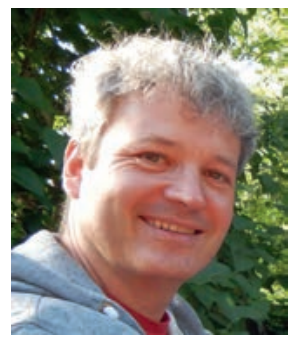

Manuel Hutterli was born in 1969 in Bern where he also grew up. He studied physics at the University of Bern, Switzerland where he graduated in 1999 with a $\mathrm{PhD}$ from the Department of Climate and Environmental Physics. After many field campaigns in Greenland and Antarctica and several years each as researcher at the University of Tucson, Arizona, USA, the University of Bern, Switzerland, and the British Antarctic Survey, Cambridge, UK, he joined TOFWERK in 2009 as a project manager in R\&D. He currently is Product Manager of the Vocus Cork Analyzer.

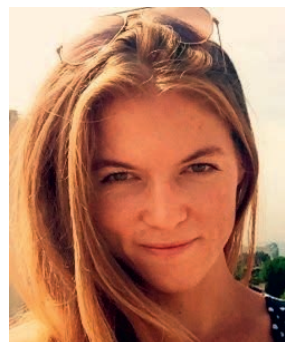

Veronika Pospisilova is an Application Scientist working at TOFWERK in Thun, Switzerland. She graduated from analytical chemistry at Charles University in Prague and obtained a $\mathrm{PhD}$ degree at the ETH Zurich in the field of atmospheric chemistry in 2019. She has extensive experience in the state-of-the art mass spectrometry for chemical analysis of aerosol and gas phase molecules. Her current research focuses on the development of chemical ionization instrumentation for realtime measurement of volatile organic compounds and its application in the field of environment monitoring and breath analysis.

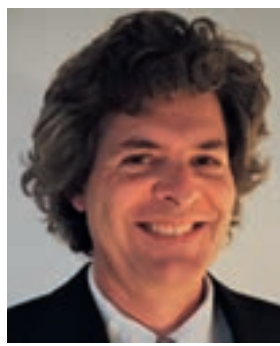

Marc Gonin is the co-founder and CEO of TOFWERK. He completed his studies in Bern, designing TOFMS for solar wind and cometary research. After his $\mathrm{PhD}$ in 1996, he worked on quality control of gases using chemical ionization mass spectrometry. He then moved to University of Giessen working as a Postdoc. From 1998 to 2002 he worked for IONWERKS in Houston, Texas.

*Correspondence: Dr. M.A. Hutterli, E-mail: hutterli@tofwerk.com, TOFWERK AG, CH-3645 Thun, Switzerland
In 2002 he founded TOFWERK with Katrin Fuhrer. Together they won the De Vigier award, and the Heinrich-Greinacher-Award. In his spare-time he is trying to nudge mass spectrometrists softly from their jargon terminology towards the standardized metrological terminology.

\section{Introduction: TOFWERK AG and TOFMS}

TOFWERK is a small- and medium-sized enterprise (SME) in Thun founded in 2002 with the initial goal to offer timeof-flight (TOF) mass analyzers as an alternative to the widely used quadrupole mass analyzers used in mass spectrometers. Mass spectrometers measure the abundance of ions of various molecular mass. In addition to the mass analyzer, they typically consist of an ion source producing electrically charged analyte ions from either gaseous, liquid, or solid samples. With the ion optics of the mass analyzer the analyte ions are transferred from the ion source via electrical and/or magnetic fields through various pressure stages to the detector with which the mass and abundance of the analyte ions are determined.

A TOF mass analyzer has several advantages over a quadrupole, especially when analyzing complex samples containing a few to several thousands of compounds of interest: Rather than filtering out one mass at a time and sequentially scanning through a list of masses like it is done with a quadrupole, an orthogonal TOF analyzer simultaneously extracts and then measures all ions of a large mass range from a continuous analyte ion beam at a very high duty cycle and at very high frequency (typically 10 to $100 \mathrm{kHz}$ ). The ions in the TOF analyzer are sorted according to their mass/charge on a flight path and a complete mass spectrum is recorded for each extraction. In addition to the fast speed, a TOFMS can also be extremely sensitive (LOD's of 10 pptv for a 1 second measurement integration) and can have a very large dynamic linear range of several orders of magnitude ( $>1 \mathrm{E} 4$ at $1 \mathrm{~s}$ measurement time). Because the mass resolving power $\mathrm{m} / \mathrm{dm}$ of a TOFMS is directly related to the length of the flight path of the ions, TOFMS of different sizes and thus mass resolving power can be designed, covering different requirements for separating isobars in complex samples. TOFWERK, for example, offers TOF analyzers with mass resolving powers ranging from 500 to 15'000 Th/Th. ${ }^{[1]}$ 
In the first years, TOFWERK was mostly selling TOF mass analyzers to OEM customers that built them into their end-user instruments and to research groups that wanted to upgrade their research instrumentation with a high-performance TOF mass analyzer. After a few years, TOFWERK started to also sell enduser TOFMS with a variety of ion sources and comprehensive data acquisition and processing software for a very diverse range of research applications. In 2017 TOFWERK USA and in 2019 TOFWERK China were incorporated to better serve these markets. In 2020 the first single-purpose industrial TOFMS solution was sold to the cork stopper industry. ${ }^{[2]}$

\section{TOFMS Requirements for Mobile Applications}

The first TOFWERK OEM customer was a leader in atmospheric research where speed, sensitivity, dynamic range, and mass resolving power are critical for continuous real-time measurements of air and aerosol composition. Such measurements are often done at remote stations or from mobile platforms, imposing challenging requirements on the design of the TOFMS in terms of robustness, flexibility, ease of use, power consumption and transportability.

The most stringent requirements must be met in mobile applications, where the instruments have to reliably acquire data when in movement and being exposed to vibration, shock, and fast changes in orientation, temperature and pressure. Often the availability of electrical power is also limited and unstable.

From the beginning TOFWERK TOFMS have thus been specifically designed to fulfil the requirements for mobile applications. In addition to a very ruggedized design of the TOF analyzer chamber, ion optics and electronics, they, e.g., also contain a custom-built turbo molecular vacuum pump that can handle the vibration and shock that typically occur during mobile applications. The product line that is most often used for mobile applications is the 'Vocus CI-TOF' which was designed for real-time chemical analysis of air based on chemical ionization (CI). ${ }^{[1]}$ These instruments are built into a frame based on standard industrial profiles allowing flexibility not only for mounting the instrument to any mobile platform but also to attach custom accessories to the instrument. Also, they can be run in any orientation. Due to the modular design and for reason of economy of scale the same ruggedized components are also built into TOFWERK product lines that are typically used in more conventional stationary applications such as research laboratories or industry (icpTOF, fibTOF, pgaTOF ${ }^{[3]}$ ).

Due to the complexity and typically higher costs of mobile applications, they are only chosen when stationary methods cannot provide the necessary information. This is, e.g., the case when samples cannot be brought to the laboratory because they are instable, or because the required time resolution is most efficiently achieved with continuous real-time measurements. For instance, ions produced naturally in the atmosphere by cosmic rays cannot be stored in containers and transported. ${ }^{[4]}$ During airplane campaigns real-time measurements with a time resolution of $1 \mathrm{~s}$ result in a spatial resolution of a couple of hundred meters. A similar spatial resolution would not be possible with discrete air sampling, which would require 3600 sample vials per flight hour.

It is worth noting that the time resolution of real-time TOFMS measurements are not normally limited by the extraction frequency of the TOF (typically $10-100 \mathrm{kHz}$ ). It is generally limited by the sensitivity towards the analyte, which is largely determined by the ion source used, or by the time response of the sample inlet and ion source for this analyte.

With the industry-leading LOD of $10 \mathrm{pptv}$ in one second, e.g. for xylene with the Vocus CI-TOF, ${ }^{[1]}$ one would have to average the measurements over a minute to resolve single digit pptv variations in the air. On the other hand, concentrations in the ppbv range or higher are resolved at $10 \mathrm{~Hz}$ or faster.
The latter, however, can only be achieved if the compounds of interest are efficiently transferred through the inlet without significantly sticking or reacting on the inlet walls which smears out fast changes in concentration. A simple, unheated inlet can lead to response times of several minutes or more for compounds with lower volatility. To optimize response times, inlets must be designed as short as possible, with a surface that minimizes sticking (e.g. PFA Teflon tubing). Most importantly, a high sample flow through the inlet to the ion source is the most efficient way to improve the response time, minimize surface interactions and wall contact memory effects.

Thus, there are conditions where the samples cannot be taken to the instrument, but the instrument must be brought to the sample. In the sections below we will present some examples of mobile TOFMS applications in which the TOFMS are operational when moved over several hundred kilometers to less than a meter.

\section{Examples of Mobile Applications 3.1 Range 10-10'000 km: Regional and Continental Scales}

Atmospheric oxidation processes have important impacts on health and climate. The atmosphere is an incredibly complex, dynamic chemical reactor in which atmospheric oxidants mix with a diverse set of natural and anthropogenic emissions generating thousands of compounds. ${ }^{[5]}$ To understand the formation and persistence of air pollution, the generation of photochemical smog and the formation of aerosol, atmospheric chemistry research and monitoring examines the chemical evolution of VOC from individual point sources up to regional and continental spatial scales.

Tracing the evolution of anthropogenic emissions emitted from urban centers and pinpointing the sources provides a unique analytical challenge, especially when combined with the diverse and inhomogeneous natural emission profiles (cities, forests, oceans). Reaction rates, emission profiles and removal processes change hourly, daily, and seasonally and have direct impacts on the oxidation products, the oxidation capacity and thus lifetime of emissions in the atmosphere.

Adding to the analytical challenges, the emissions and reaction products are also rapidly dispersed and diluted across large spatial scales by ventilation, removed by chemical conversion, scavenged by wet or dry deposition, and partition between the gas and particulate phase with rates and direction depending on temperature, humidity, and dilution.

Given the spatial scale over which emissions and oxidation take place in the atmosphere, ground-based measurements often require many measurement locations to be arranged in a network to provide adequate spatial resolution. Such networks are generally unable to capture the total effects of simultaneous dilution and chemical reaction, especially across all three spatial dimensions.

Airborne measurements involve the deployment of CI-TOF instruments aboard aircraft which allow excellent spatial sample coverage from altitudes just above earth's surface through the troposphere and into the lower stratosphere. Instruments are usually installed in specialized flight racks and sample air from outside the aircraft through specialized window plates or other aerodynamic inlets (Fig. 1). Aircraft platforms provide a unique approach to address emissions and oxidation across large spatial scales, for example following outflow from major urban centers, natural terrestrial and oceanic emissions. Recent examples of research aircraft campaigns have targeted a number of important atmospheric chemistry topics over the last decade relating to air quality and climate.

The first aircraft deployment of a CI-TOF was as part of the NOAA SENEX campaign in 2013.[6] Researchers from the University of Washington in collaboration with a team from NOAA ESRL deployed a CI-TOF on a WP-3D aircraft and sur- 

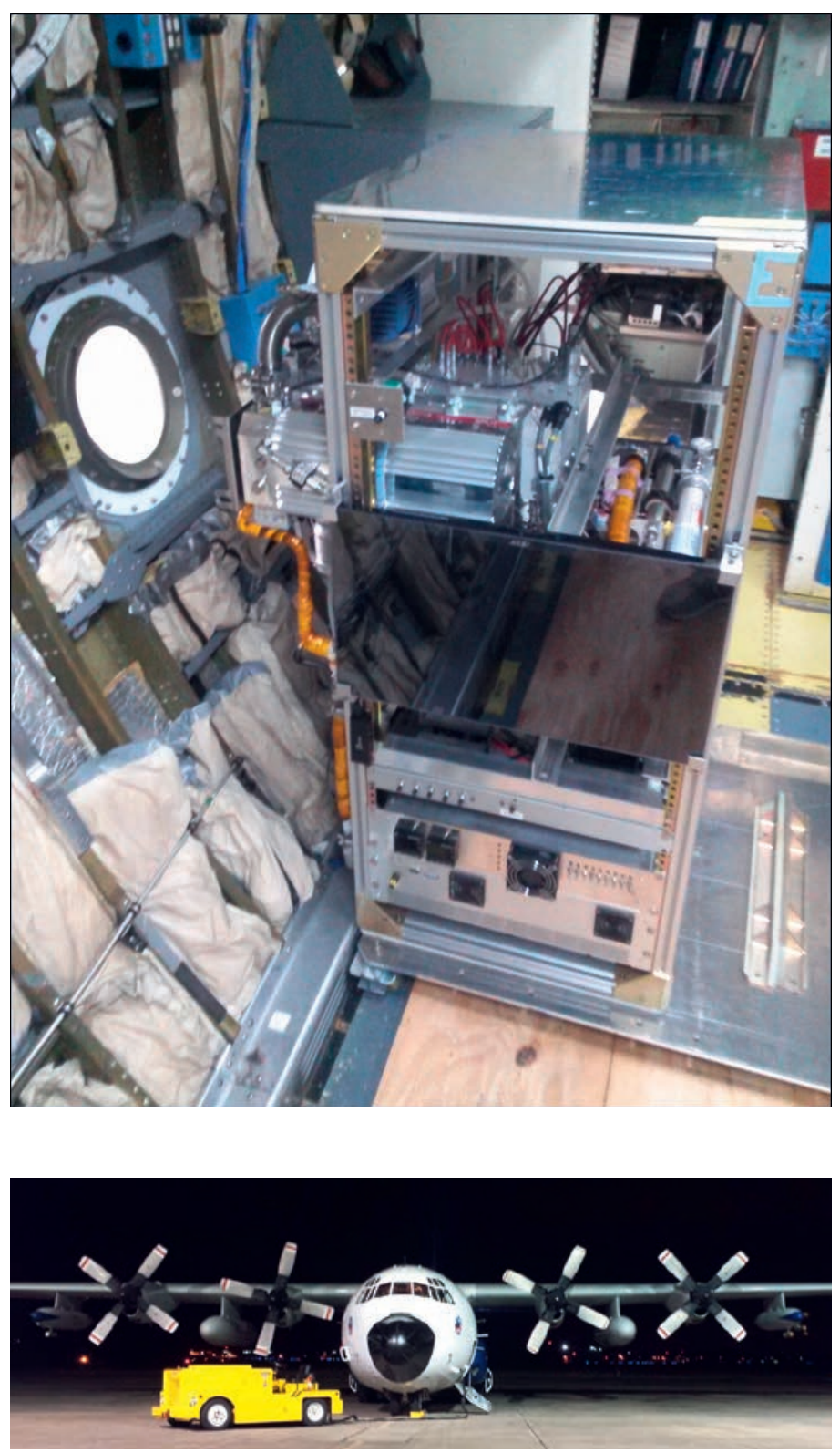

Fig. 1. Top: A Vocus Cl-TOF mounted in a special flight rack in a $\mathrm{C}-130$ Hercules research aircraft (bottom) sampling outside air.

veyed the interaction of biogenic and anthropogenic compounds over the Southeastern United States during summertime. ${ }^{[7]}$

Following this successful deployment, CI-TOF instruments have flown on every major atmospheric chemistry aircraft campaign to provide unparalleled chemical characterization of air around the world.

CI-TOF deployments aboard research aircraft in the last decade have become routine but critical parts of the scientific payload. Campaigns during the summer months, where photochemistry is most active have utilized CI-TOF instruments to survey diverse sources and chemical systems including the impact of wildfires on the local and regional air quality and climate, ${ }^{[8]}$ and emissions over forests and grasslands. ${ }^{[9,10]}$ During winter, the reduction in sunlight and cold temperatures has important impacts on the oxidation pathways and lifetime of pollutants in the atmosphere. Research topics addressed by airborne instruments include lifetime of the urban outflow off the east coast of the United States, the evolution of powerplant plumes extending over urban and rural areas, the generation of halogen radicals through multiphase chemistry; all of which benefit from the comprehensive chemical coverage afforded by CI-TOF instruments. Even global chemical snapshots have been produced by flying aircraft around the world multiple times with regular vertical profiling.[11]

\subsection{Range 0.1-10 km: Fenceline Monitoring}

In the regulatory world, a mobile laboratory equipped with real-time trace gas detectors can create maps that show chemical abundances, emission sources, and transport in a city or a larger region. This information can be used to identify areas with environmental problems, assess whether businesses are complying with legal regulations, and provide useful information to the public.

The Vocus Elf (nominal mass resolving power $500 \mathrm{Th} / \mathrm{Th}^{[1]}$ ) is able to measure many species important to regulators, such as BTX (benzene, toluene, xylene), solvents, and ozone precursors. The size, price-point, and power requirements allow regulatory agencies to deploy Vocus Elfs in vehicles.

In the example shown here, a Vocus Elf using $\mathrm{H}_{3} \mathrm{O}^{+}$ion chemistry was installed in the rear seat of an electric passenger car. The instrument was run entirely off the car battery, and no special modifications were made to the car. The instrument in the car is shown in the inset in Fig. 2.

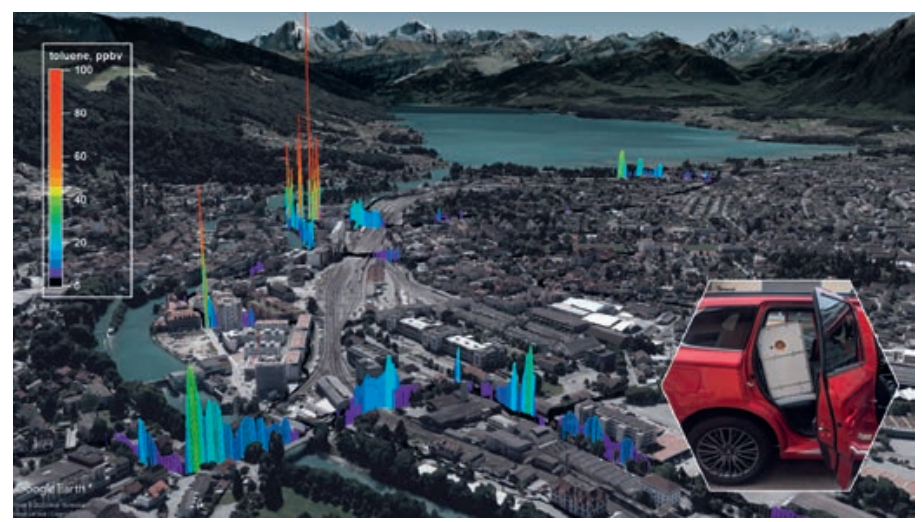

Fig. 2. Aerial picture of Thun, Switzerland with the measured toluene concentrations along the drive route. The insert on the lower right shows the Vocus Elf Cl-TOF on the backseat of the car.

The instrument was connected to a sampling probe that extended over the car windshield. A 90-minute drive was conducted through the city of Thun, entirely on electric power, so that no emissions from the mobile lab would be observed. During the drive, important locations were targeted, including major streets, an official air quality monitoring station, and some point sources such as gas stations, the train station, and a water treatment plant.

Because the instrument reports measurement in real time, VOC hotspots can be seen by the operator during the drive. Fig. 2 reveals some of these hotspots. It shows the concentration of toluene along the drive path, and high concentrations of this VOC can be seen near intersections, the train station, major roads, and gas stations. Some of the hotspots had very high concentrations (more than $100 \mathrm{ppbv}$ ). Especially high concentrations of aromatics (BTX) were measured along a large construction zone where fresh asphalt was being prepared. Stationary monitoring stations often miss large sources such as these, which can be temporary and located far away from the official measurement station.

While just one species (toluene) is shown in Fig. 2, more than 100 different species were actually measured during each second of the drive. By investigating the correlations between these different species, different point sources can be distinguished and identified based on their chemical profile. Fig. 3 shows some of the different groups of compounds that were identified by correlation analysis. These groups include two different sources related 


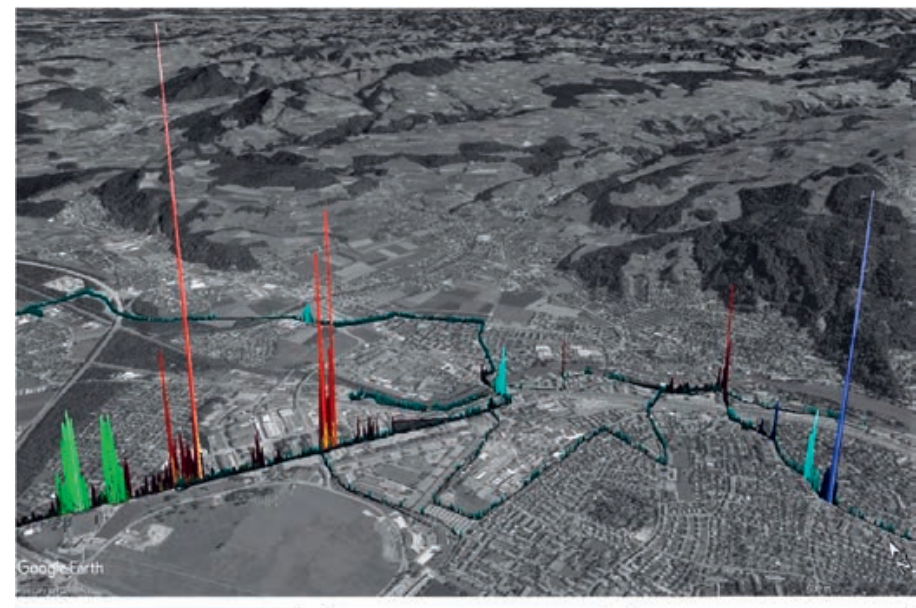

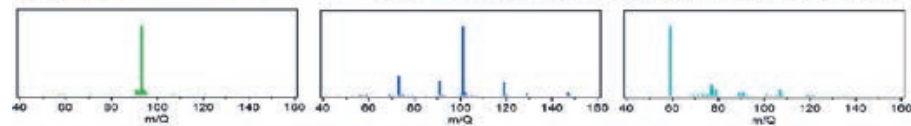

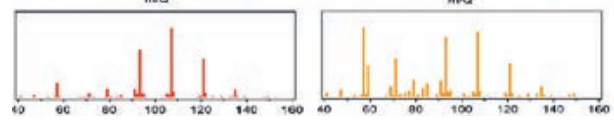

Fig. 3. Distinct VOC sources detected in Thun along the drive route of the mobile laboratory, with colour and intensity indicating the identity and relative abundance of each source. The smaller panels show the mass spectral VOC fingerprint of each source.

to vehicle traffic, intense sources of toluene and acetone, and a source containing some aldehydes, which might have been solvents from a medical clinic.

\subsection{Range 5-100 m: Indoor Air Quality Monitoring and Breath Analyses}

\subsubsection{Real-time Measurement of Indoor VOCs}

Real-time monitoring of VOCs in the working environment has become more prominent in recent decades. With the majority of people spending most of their time indoors poor indoor air quality can affect people's health, comfort, as well as work performance. Industrial processes, construction materials or even personal care products can be a source of a diverse set of VOCs. ${ }^{[12,13]}$ These can accumulate within buildings, especially in areas with insufficient ventilation. This can lead to adverse health effects and to contamination of sensitive working environments. Indoor air pollution is acknowledged as one of the top risks to public health by the world health organization. ${ }^{[14]}$ Therefore, it is crucial to properly understand and identify the pollutant sources in order to control the human exposure to potentially dangerous levels of indoor air pollutants.
TOFWERK Vocus CI-TOF provide a simple, sensitive, and fast way to evaluate indoor air quality quantitatively. They enable online measurements of most emerging contaminants without the need for extensive libraries and without pre-separation. They are mobile and can be deployed rapidly throughout buildings to provide a survey of air quality and identify and pinpoint sources of chemicals. Longterm stationary monitoring measurements can reveal daily patterns in indoor air that are related to ventilation and activities which release VOCs into the air such as cooking, industrial processes, cleaning or accidental release of chemicals via spills or leaks.

Fig. 4 shows the results of screening an industrial area for VOC pollutants with a mobile Vocus CI-TOF in less than 30 minutes by moving the instrument into the different areas.

The concentrations are shown on a log scale and can vary by a factor of 5-10 depending on the location. The rooms with active ventilation (e.g. chemical lab) showed lowest VOC levels, comparable to ambient air. The cleaning room showed high levels of propanol coming presumably from the cleaning and disinfectant chemicals stored inside. The highest concentration of VOCs in general was measured in the clean room which was under construction at the time of measurement.

The possibility of switching between the $\mathrm{H}_{3} \mathrm{O}^{+}$ionization mode (traditional proton transfer reaction, 'PTR' mode) and the $\mathrm{NH}_{4}^{+}$ ionization mode ${ }^{[1]}$ allows increasing the number of detected compounds. Fig. 5 shows an example of indoor air mass spectra measured by the Vocus in $\mathrm{NH}_{4}^{+}$mode.

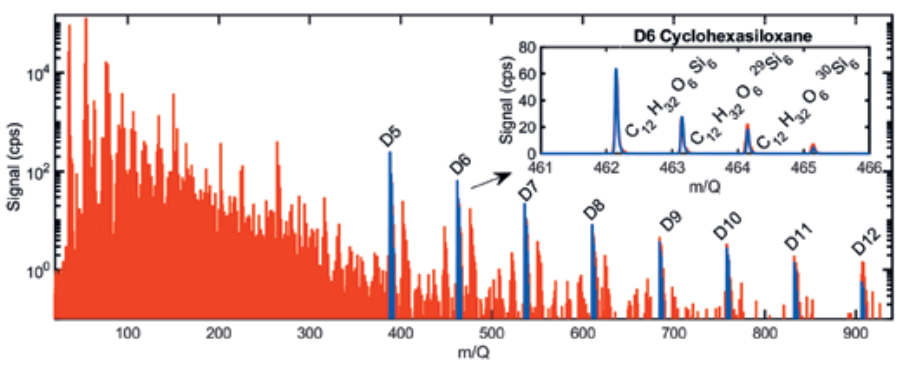

Fig. 5. Mass spectrum of indoor air measured by the Vocus Cl-TOF in $\mathrm{NH}_{4}{ }^{+}$mode. Series of siloxanes are highlighted in blue. In the inset picture the specific isotope pattern of D6 (cyclohexasilioxane) is highlighted.

In this ionization scheme the typical series of siloxanes ranging from D5 (dodecamethylcylcopentasiloxane) to up to D12 (dodecamethylcyclohexasiloxane) was detected. Siloxanes are stable chemicals persistent in the environment that are widely used in plastics, lubricants, oils and cosmetics. The most intense siloxane observed was D5 which is a common compound
Mobile Indoor Air quality monitoring

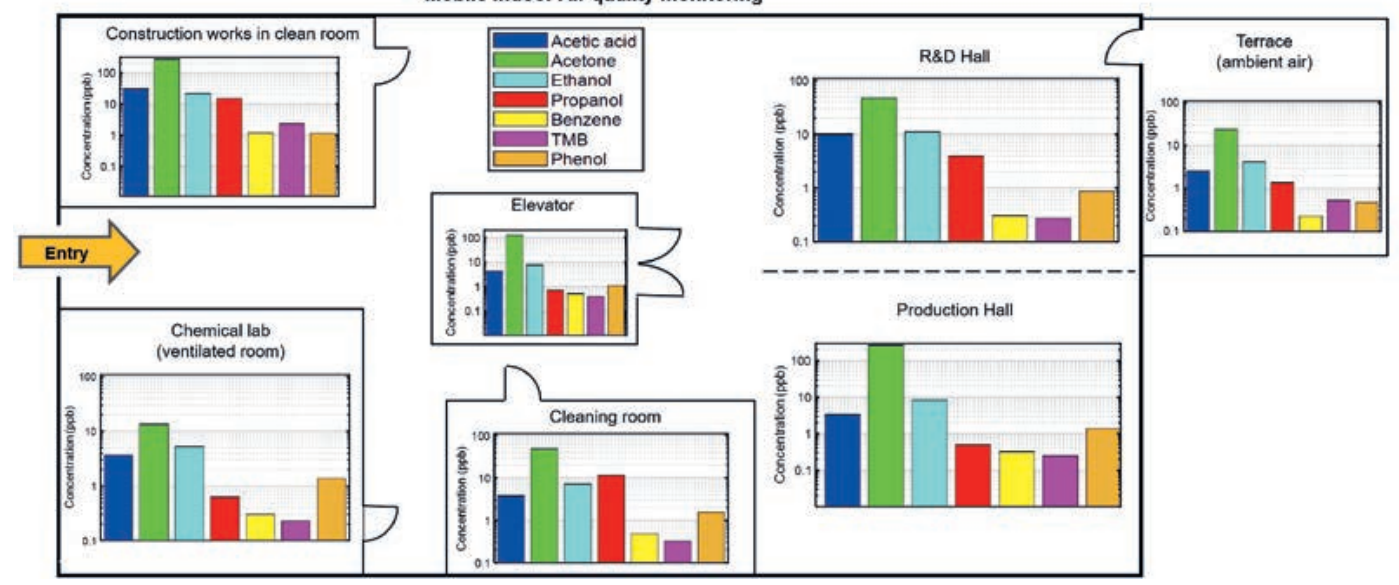

Fig. 4. Concentration of selected VOCs measured while moving the Vocus Cl-TOF between areas in the industrial building. 
found in urban air and associated with the use of personal care products.

\subsubsection{Breath Analyses: COVID-19 Detection}

Exhaled breath contains hundreds of volatile metabolites reflecting the biochemical processes occurring in the human body. These molecules are often transported from the blood into the airways via alveolar gas exchange in the lungs and as such offer a unique way to investigate human metabolism, study pharmacokinetic pathways, individual response of pharmacological treatment or can serve as disease specific biomarkers. The chase for specific metabolites that are altered with certain disease conditions has been the target of many breath studies in the past. ${ }^{[15,16]}$ Indeed, establishing molecular fingerprints of human health would offer unprecedented diagnostic tools with numerous advantages and holds a big potential in the field of clinical diagnosis. In comparison to traditional clinical sampling techniques, breath analysis is a simple, fast, and noninvasive sampling approach with virtually unlimited availability of sample. When performed in real time the results can be directly available for the clinical personnel. However, despite the numerous studies and promising results towards identifying molecular patterns in exhaled breath, no widespread application of breath tests is seen in the daily clinical environment. The reasons for this are likely due to the lack of standardized sampling approaches, the variety of analytical platforms used, limited data for statistical analysis, lack of understanding of the biological origin of the identified VOCs and missing follow-up validation studies of preliminary results. ${ }^{[15]}$

Breath presents a challenging analytical sample due to its high humidity and trace levels of the target molecules. Fast quantitative analysis is preferred as it allows the detection of rapid changes in the concentration of the volatile organics. Further, real-time measurements also avoid sample collection or its preparation which can introduce errors in the process of the analysis. TOFWERK has recently introduced the Vocus Breath Analyzer (VBA), which is an instrument offering intuitive, reproducible and humidity independent analysis of breath exhalations with unprecedented sensitivity.

VBA consists of a Vocus CI TOFMS, high-end breath inlet and software with automatic breath detection. The breath is sampled directly into the instrument and ionized by the reaction with reagent ions $\left(\mathrm{H}_{3} \mathrm{O}^{+}\right.$or $\left.\mathrm{NH}_{4}^{+}\right)$. Due to the characteristics of the Vocus ion source, the response is not affected by water vapor which makes the measurement quantitative under high humid conditions. All VOCs in the exhalation are detected simultaneously and in real time. The

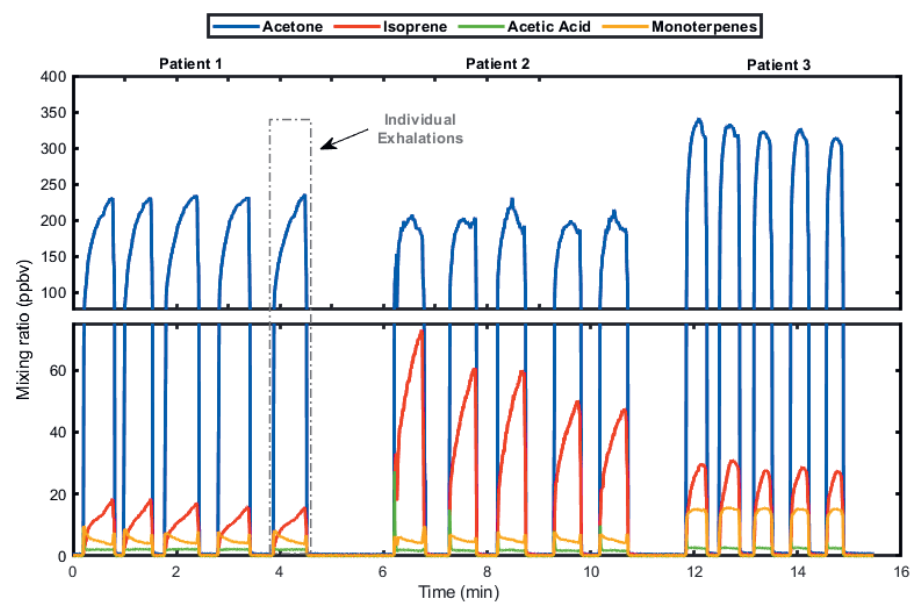

Fig. 6. Example of sets of five exhalations by three patients measured with the Vocus Breath Analyzer. Time traces of acetone, isoprene, acetic acid and the sum of a group of monoterpenes are selected and displayed as mixing ratios in ppb (parts-per-billion) units. breath inlet provides a heated, optimized interface for reproducible sampling of human breath with a valve avoiding re-inhalation to ensure no risk of cross-contamination and condensation of low-volatile organics. In Fig. 6 an example of sets of five exhalations by three patients measured with the VBA is shown. The mixing ratios depicted in the figure are of acetone, isoprene, acetic acid and the sum of a group of monoterpenes.

The COVID-19 pandemic has had devastating effects worldwide in terms of life loss, public health, and economy. To prevent further spreading of the virus in the coming years, vaccination and massive testing remain essential. Currently the diagnosis is based on reverse transcription-polymerase chain reaction (RT-PCR) where a sample from a nasal swab is tested for the presence of ribonucleic acid (RNA) of the SARS-CoV-2. This technique requires lengthy procedures and is hardly applicable for fast screening. Tests allowing rapid diagnosis such as antigenic tests suffer from fairly poor performance with sensitivity close to $60 \%$ in asymptomatic individuals. ${ }^{[17]}$ To address this gap the researchers from Ircelyon conducted two clinical trials between 2020 and 2021, deploying the mobile VBA in the Croix Rousse Hospital and in the Gerland center in Lyon which temporarily served as a COVID screening point (Fig.7).

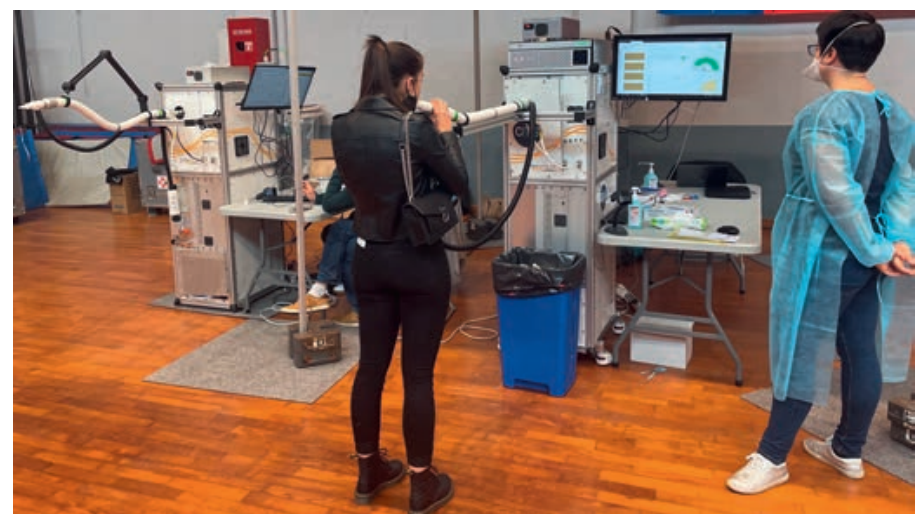

Fig. 7. Deployment of two Vocus Breath Analysers to the Gerland center in Lyon. The VBA on the left was deployed by the Laboratory of Atmospheric chemistry from the Paul Scherrer Institute in Switzerland to obtain data for repeatability of the method. One thousand individuals performed exhalations into both instruments. The VBA on the right was equipped with an uninterruptible power supply unit (UPS) and wheels which allowed its displacement among various hospital rooms and departments.

During the clinical trial at the Croix Rousse Hospital the VBA could be deployed to any site of the hospital within a short period of time, including clinical examination rooms or intensive care units. The mobile VBA was wheeled to the patients to do the breath analyses directly at their beds, so that they did not have to be displaced (see e.g. news coverage in ref. [18]). The VBA was also used to passively sample exhaled air from respirators of COVID-19 patients in the intensive care units (Fig. 8).

The goal of the study was to develop a diagnostic method based on breath analysis for population screening allowing instant identification of specific breath biomarkers of this highly infectious virus. Such a method would avoid the need for uncomfortable sampling procedures while providing basically real-time results. The individuals taking part in the study underwent nasopharyngeal sampling and afterwards were asked to participate in the study. The breath of the volunteers was analyzed directly via VBA while the RT-PCR results of the nasopharyngeal swab served as a reference. The real-time nature of the VBA allowed the measurement of one person per minute. The measurement data will be used to develop a model based on machine learning techniques for a COVID-19 breath screening test. 


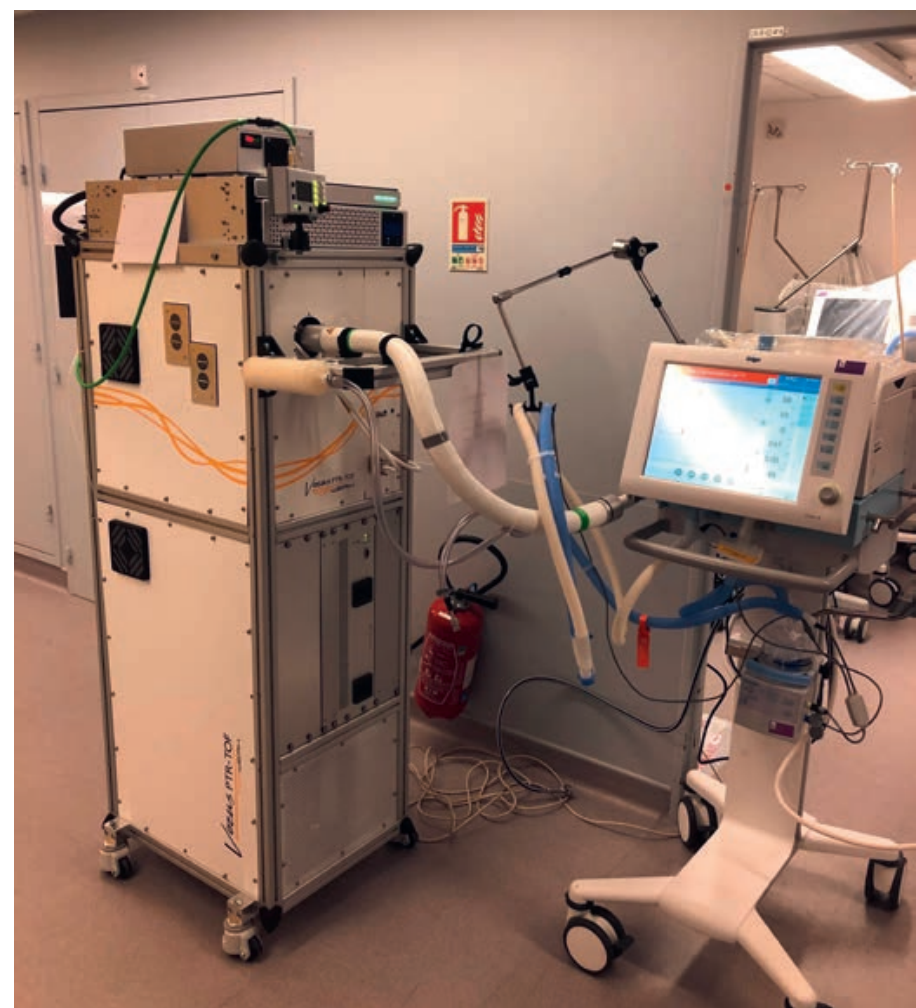

Fig. 8. The mobile VBA in an intensive care unit is passively sampling exhaled air from a respirator of a COVID-19 patient. Photo: CNRS and $\mathrm{HCL}$.

\subsection{Range 0.5-5 m: Autosamplers}

\subsubsection{Plant Autosampler}

Autosamplers typically consist of an automated mechanism that either moves a sample vial to a sampling position where the sample is extracted for subsequent analyses or a sample extractor (normally a syringe) is automatically moving to a sample vial in a sample tray to sequentially extract the analyte and transfer it through a tubing to the analyzer. However, this is not always possible, if e.g. the samples are too large and/or cannot be moved and the required time resolution and/or limited sample volume prevents the use of long sample transfer lines.

This was, for example, the case in an application of the Institute of Plant Sciences (IPS), University of Bern, Switzerland (Prof. M. Erb) where the time evolution of VOCs in glass containers containing plants was to be recorded at high temporal resolution. The interactions of plants with their environment determines their survival in nature and yields in agriculture. Plants can release specific VOCs to attract beneficial organisms and can detect VOCs as environmental cues. ${ }^{[19]}$ Time-resolved monitoring of VOC exposure and emissions for large numbers of individual plants is critical for understanding the dynamic roles and mechanisms of VOC-dependent plant-environment interactions.

In collaboration with IPS and industrial partners (Abon Life Sciences and Bibus AG) we developed an autosampler specifically for this purpose. The plant autosampler had to include 102 quartz glass containers of a few liters volume that are purged with clean or doped air to investigate $e . g$. the reaction of plants to VOCs or to simulated herbivore attack in dynamic headspace measurements. Due to the continuous purging and the size of the glass containers it was not practical to automatically transport the containers to a sampling position. Further, the sample air consumption had to be minimal $(\sim 100 \mathrm{sccm})$ and time response fast $(<100 \mathrm{~ms})$. This prevented the use of a several meter-long sample transfer line moving between the containers, which would be done in a conventional autosampler setup.

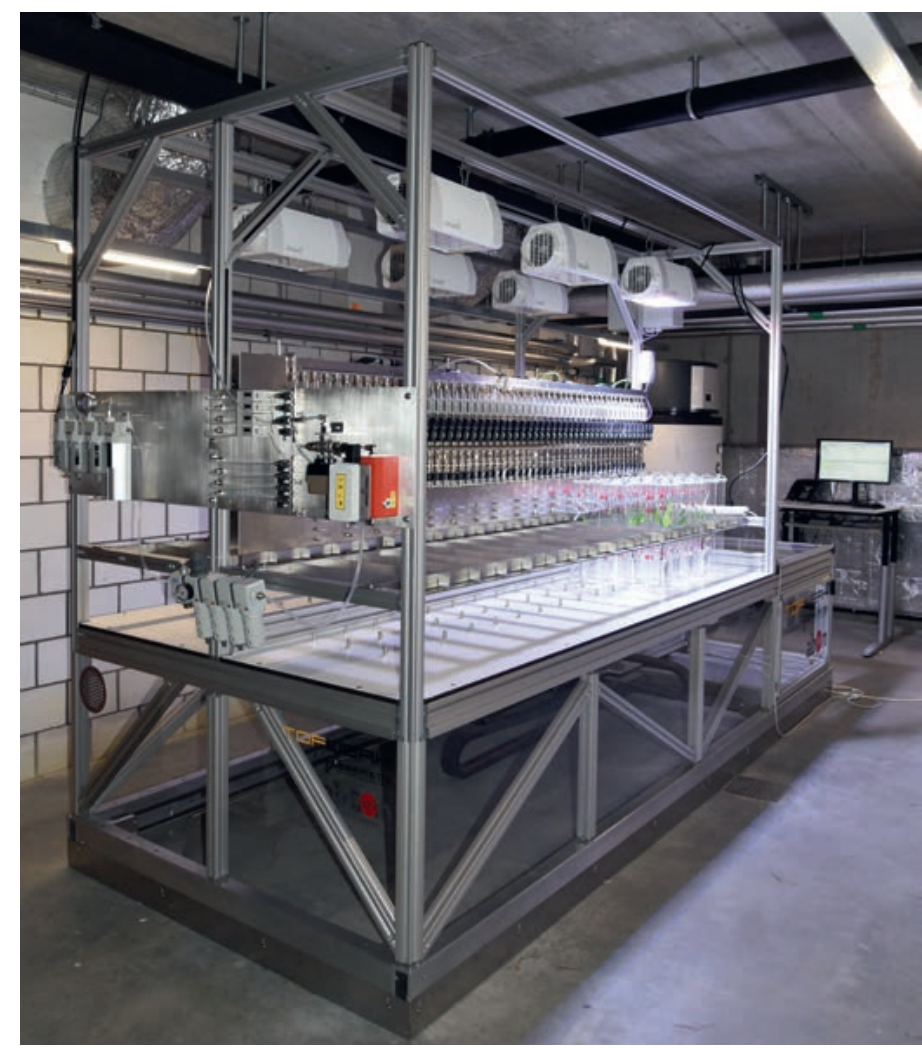

Fig. 9. Plant autosampler. The Vocus S Cl-TOF is moving under the table among the docking ports of the plant containers on the table, sampling their purge gas flow for real-time VOC analyses.

Thus the autosampler (Fig. 9) was designed such that a Vocus $\mathrm{S}$ (nominal mass resolving power $5000 \mathrm{Th} / \mathrm{Th}$ ) is moved by up to about $3 \mathrm{~m}$ under the table with the plant containers. It is facing with the inlet upwards and is sequentially collecting air samples from the containers purge flow docking ports through a short $(10 \mathrm{~cm})$ heated inlet (a movie of the autosampler in action is available online ${ }^{[20]}$ ).

A typical sample protocol consists of a 10-second measurement of sample air from a plant followed by a 5-second zero air measurement while the TOFMS is transferred to the next position. The zero air measurement purges the inlet between samples and provides a clear separation of the data of different plants (Fig. 10).
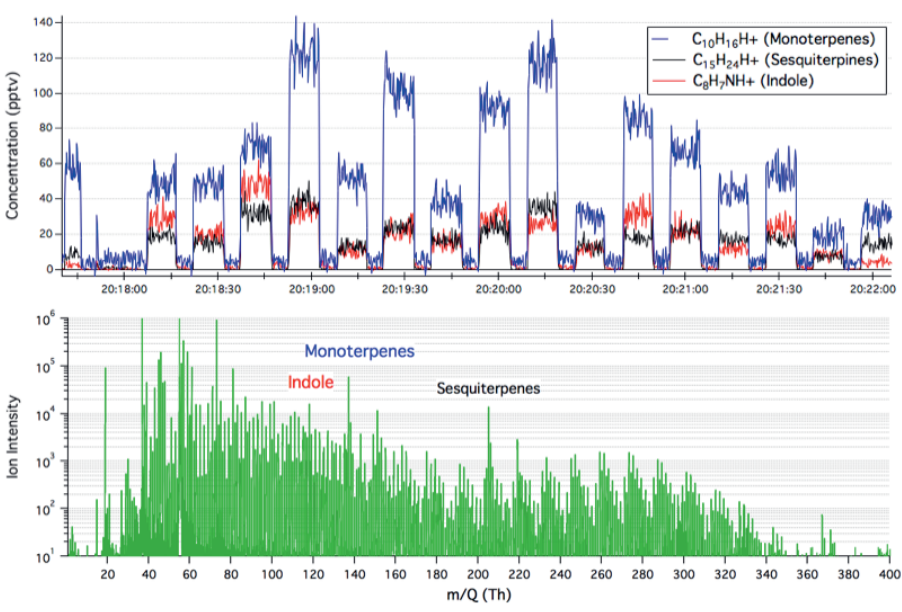

Fig. 10. Top: An example of an autosampler cycle measuring a series of 17 plants. Concentrations of select VOCs are plotted at $5 \mathrm{~Hz}$ ( 5 unique mass spectra per second). In this case, 17 individual plants were sampled with a measurement time of only 10 seconds per plant. Between individual plants, a fast zero air measurement is performed for 5 seconds. Bottom: An example mass spectrum (log scale) from a single plant averaged for the 10-second sample measurement time. 
The autosampler is capable of resolving individual plant kinetics for up to 102 plants with a time resolution below 30 minutes or accordingly faster for a smaller set of plant containers. The $5 \mathrm{~Hz}$ time-series in Fig. 10 reveals the excellent signal-to-noise of the Vocus-S even at concentrations approaching single digit pptv concentrations and a response time of the instrument in the order of $100 \mathrm{~ms}$ or better even for sticky compounds such as terpenes. Fig. 10, bottom, shows an example of a mass spectrum averaged over 10 seconds showing that hundreds of compounds are detected and monitored in seconds.

In an experiment on 17 individual maize plants the attack from Spodoptera littoralis larvae, which eat maize leaves, was simulated and the plants' kinetic response was monitored over 10 hours. The emission kinetics of selected VOCs over time after the simulated insect attack on one of the plants is depicted in Fig. 11.

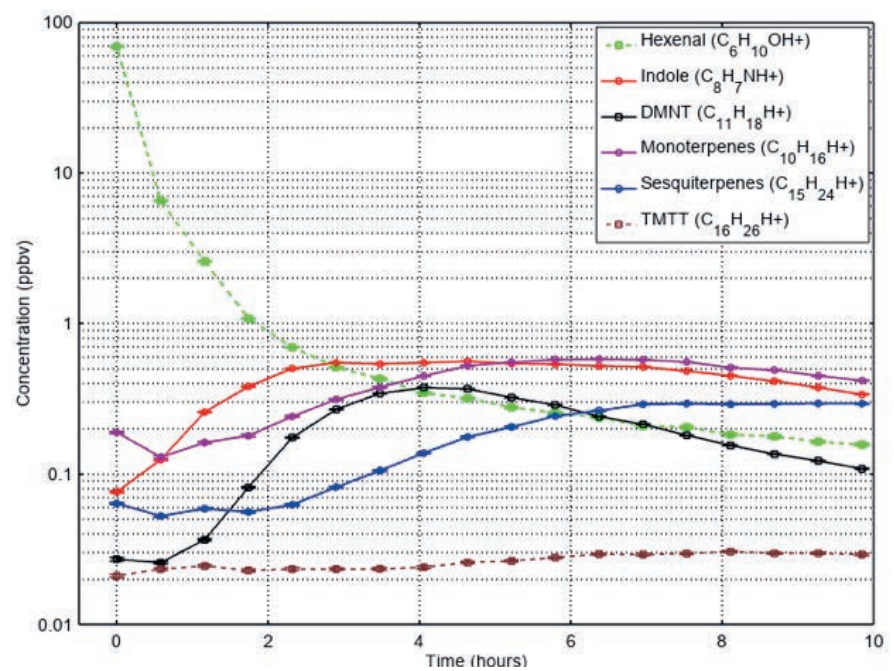

Fig. 11. The emission kinetics of selected VOCs over time after a simulated insect attack of one of the plants in Fig. 10.

Initially, green leaf volatiles such as hexenal are rapidly emitted after the leaves are scratched and exposed to insect regurgitate. After 30 minutes, indole starts to be released as a priming cue that can induce defenses in neighboring plants. Terpenes, which can attract predators of herbivores, are released at later points in time.

The quasi-simultaneous sampling of over 100 plants using one mass spectrometer provides a unique solution for systematic studies of a large number of samples, thus allowing for genetic mapping and large-scale comparative approaches in plant science and other fields. Such an autosampler fitted e.g. with small climate chambers instead of the plant containers could be used to determine the temporal evolution of VOC emissions from building-, car manufacture, or other materials at various environmental conditions, often required by Health and Safety regulations.

\subsubsection{Vocus Cork Analyzer (VCA)}

40 years ago, a research team at the Swiss Federal Research Station Wädenswil identified 2,4,6-trichloroanisole (TCA) as the main culprit for the unpleasant, musty cork taint sometimes found in bottled wine, ${ }^{[21]}$ which causes huge economic losses to the wine industry. The human sensory threshold for TCA is extremely low, already 1-2 ng/L can be perceived as tainted. TCA cannot be completely removed from natural cork stoppers without damaging the structural and mechanical properties of the cork. The origin of the TCA in cork oak bark is debated ${ }^{[22]}$ and it is very inhomogeneously distributed within the bark. Therefore, only a non-destructive and fast TCA screening of single cork stoppers can reliably guarantee corks with TCA levels below the sensory threshold without discarding large amounts of good bark. The VCA (Fig. 12, $3.31 \times 2.45 \times 2.00 \mathrm{~m}$ ) developed by TOFWERK and an industrial partner (LRP, Thun, Switzerland) is the first industrial solution of an on-line quantification of TCA in single cork stoppers below the sensory threshold in only 3 seconds. ${ }^{22]}$

The VCA consists of a pre-heating oven in which about 350 corks are sequentially preheated to $120^{\circ} \mathrm{C}$ to increase the release of potential TCA (Fig. 12). The corks are then transferred into individual sample cavities of an autosampler ring where the thermally desorbed TCA is accumulated for a couple of minutes in a dynamic headspace setup. The headspace air is then transferred to a Vocus 2R with a mass resolving power of up to $15^{\prime} 000 \mathrm{Th} /$ Th and analysed for TCA and other off-flavours using soft and selective $\mathrm{NO}^{+}$chemical ionization. ${ }^{23]}$ The sampling, TCA quantification, and sorting of the corks is completely automated and a VCA can screen and sort more than 20'000 corks/day or 6'000'000 corks/year. In this application the Vocus $2 \mathrm{R}$ is mounted on its side on a cart so it can be easily turned away from the cork autosampler for a short daily maintenance of the transfer line and the sample air filter exchange without having to turn the instrument off. This industrial solution is our mobile application in which an operating TOFMS is moved the least, by less than a meter (a movie of the VCA in action is available online ${ }^{[23]}$ ).
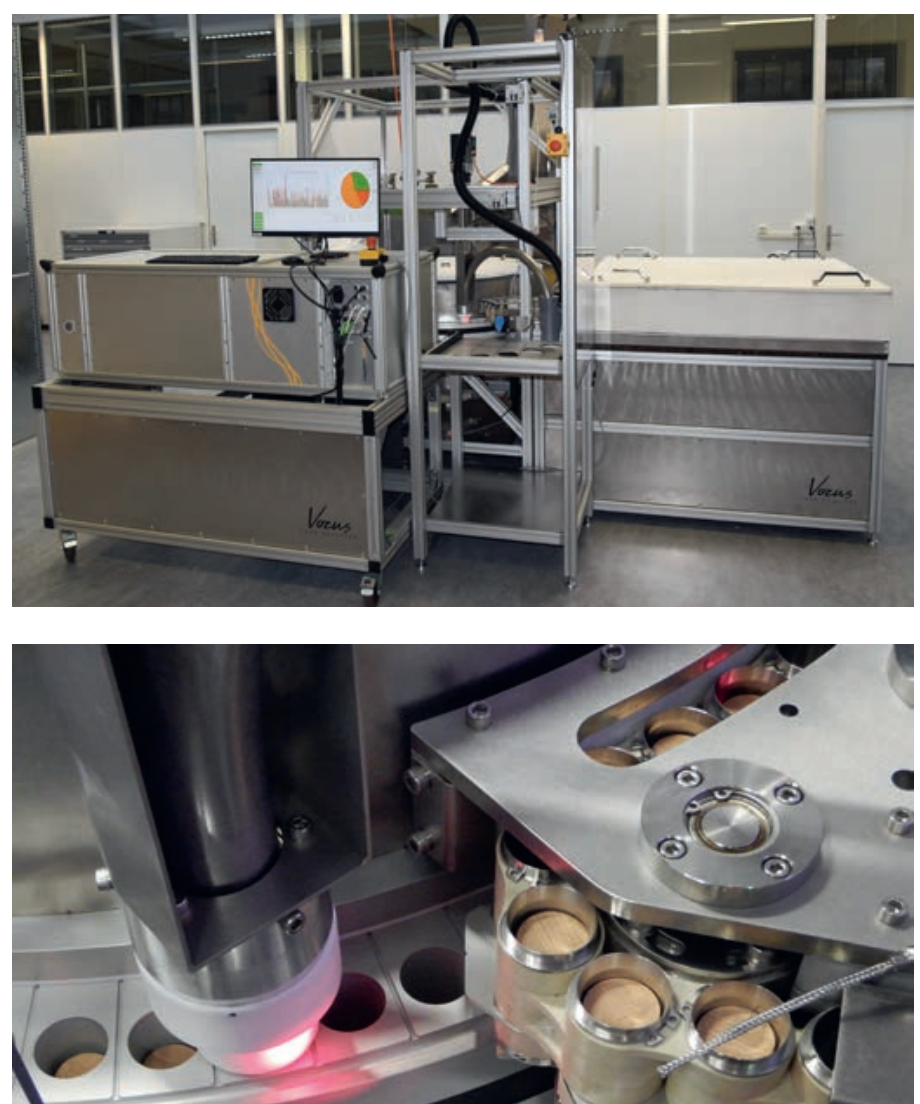

Fig. 12. Top: The Vocus Cork Analyzer (VCA) with the cork oven on the right (white cover), the cork sorting tower in the centre and the Vocus-2R (with yellow lines) on the left in horizontal position on a movable cart. Not visible is the autosampler ring behind the TOFMS. Bottom: picture of preheated corks in the oven chain (right) before they are transferred into the cavities of the autosampler ring in which the thermal desorption dynamic headspace measurements are done after an equilibration time of about 2 minutes. On the left: corks in the autosampler ring cavities after analyses and before being removed by the sorter arm (white block). Every 3 seconds a cork is loaded into the VCA and about 20 minutes later sorted into one of 5 bins according to its TCA content. 


\section{Conclusions}

The majority of the TOFWERK TOFMS are used in stationary applications. However, the unique possibility to also operate them in the harsh and demanding environments encountered during mobile applications has proven their feasibility for a wide range of new applications. This was not only demonstrated for different geographical scales, but also for vastly different research applications, regulatory analyses and recently also industrial applications with the Vocus Cork Analyzer. The demand for such challenging TOFMS applications has led to many diverse and exciting $\mathrm{R} \& \mathrm{D}$ projects carried out in close collaboration with our partners. This is to our mutual benefit; while continuously adapting and improving the TOFMS technology to the emerging needs, we can provide unique analytical instrumentation and solutions for a growing number of users in research, industry and regulatory authorities.

\section{Acknowledgements}

We would like to thank Prof. Dr. Renato Zenobi for giving us the opportunity to present our company history and research in CHIMIA and an anonymous reviewer for valuable comments. We acknowledge support by Dr. Abigail Koss and Dr. Felipe Lopez-Hilfiker.

Received: October 31, 2021

[1] Vocus CI-TOF - Online Detection of VOCs, https://www.tofwerk.com/products/vocus/, accessed October 30, 2021.

[2] Vocus Cork Analyzer - Industrial solution for cork off-flavor analysis, https://www.tofwerk.com/products/vocus-cork-analyzer/, accessed October 30, 2021.

[3] TOFWERK Time-of-Flight Mass Spectrometers, https://www.tofwerk.com/products/, accessed October 30, 2021

[4] F. Bianchi, J. Tröstl, H. Junninen, C. Frege, S. Henne, C. R. Hoyle, U. Molteni, E. Herrmann, A. Adamov, N. Bukowiecki, X. Chen, J. Duplissy, M. Gysel, M. Hutterli, J. Kangasluoma, J. Kontkanen, A. Kürten, H. E. Manninen, S. Münch, O. Peräkylä, T. Petäjä, L. Rondo, C. Williamson, E. Weingartner, J. Curtius, D. R. Worsnop, M. Kulmala, J. Dommen, U. Baltensperger, Science 2016, 352, 1109, https://doi.org/10.1126/science.aad5456.

[5] A. H. Goldstein, I. E. Galbally, Environ. Sci. Technol. 2007, 41, 1514, https://doi.org/10.1021/es072476p.

[6] B. H. Lee, F. D. Lopez-Hilfiker, C. Mohr, T. Kurtén, D. R. Worsnop, J. A. Thornton, Environ. Sci. Technol. 2014, 48, 6309, https://doi.org/10.1021/es500362a.

[7] C. Warneke, M. Trainer, J. A. de Gouw, D. D. Parrish, D. W. Fahey, A. R. Ravishankara, A. M. Middlebrook, C. A. Brock, J. M. Roberts, S. S. Brown, J. A. Neuman, B. M. Lerner, D. Lack, D. Law, G. Hübler, I. Pollack, S. Sjostedt, T. B. Ryerson, J. B. Gilman, J. Liao, J. Holloway, J. Peischl, J. B. Nowak, K. C. Aikin, K.-E. Min, R. A. Washenfelder, M. G. Graus, M. Richardson, M. Z. Markovic, N. L. Wagner, A. Welti, P. R. Veres, P. Edwards, J. P. Schwarz, T. Gordon, W. P. Dube, S. A. McKeen, J. Brioude, R. Ahmadov, A. Bougiatioti, J. J. Lin, A. Nenes, G. M. Wolfe, T. F. Hanisco, B. H. Lee, F. D. Lopez-Hilfiker, J. A. Thornton, F. N. Keutsch, J. Kaiser, J. Mao, C. D. Hatch, Atmos. Meas. Tech. 2016, 9, 3063, https://doi.org/10.5194/amt-9-3063- 2016.

[8] B. B. Palm, Q. Peng, C. D. Fredrickson, B. H. Lee, L. A. Garofalo, M. A Pothier, S. M. Kreidenweis, D. K. Farmer, R. P. Pokhrel, Y. Shen, S. M. Murphy, W. Permar, L. Hu, T. L. Campos, S. R. Hall, K. Ullmann, X. Zhang, F. Flocke, E. V. Fischer, J. A. Thornton, Proc. Natl. Acad. Sci. USA 2020 117, 29469, https://doi.org/10.1073/pnas.2012218117.

[9] J. D. Fast, L. K. Berg, L. Alexander, D. Bell, E. D’Ambro, J. Hubbe, C. Kuang, J. Liu, C. Long, A. Matthews, F. Mei, R. Newsom, M. Pekour, T. Pinterich, B. Schmid, S. Schobesberger, J. Shilling, J.
N. Smith, S. Springston, K. Suski, J. A. Thornton, J. Tomlinson, J. Wang, H. Xiao, A. Zelenyuk, Bull. Am. Meteorol. Soc. 2019, 100, 821, https://doi.org/10.1175/BAMS-D-18-0030.1

[10] B. H. Lee, F. D. Lopez-Hilfiker, P. R. Veres, E. E. McDuffie, D. L. Fibiger, T. L. Sparks, C. J. Ebben, J. R. Green, J. C. Schroder, P. Campuzano-Jost, S Iyer, E. L. D'Ambro, S. Schobesberger, S. S. Brown, P. J. Wooldridge, R. C. Cohen, M. N. Fiddler, S. Bililign, J. L. Jimenez, T. Kurtén, A. J. Weinheimer, L. Jaegle, J. A. Thornton, J. Geophys. Res. Atmos. 2018, 123, 7670, https://doi.org/10.1029/2017JD028082.

[11] P. R. Veres, J. A. Neuman, T. H. Bertram, E. Assaf, G. M. Wolfe, C. J. Williamson, B. Weinzierl, S. Tilmes, C. R. Thompson, A. B. Thames, J. C. Schroder, A. Saiz-Lopez, A. W. Rollins, J. M. Roberts, D. Price, J. Peischl, B. A. Nault, K. H. Møller, D. O. Miller, S. Meinardi, Q Li, J.-F. Lamarque, A. Kupc, H. G. Kjaergaard, D. Kinnison, J. L. Jimenez, C. M. Jernigan, R. S. Hornbrook, A. Hills, M. Dollner, D A. Day, C. A. Cuevas, P. Campuzano-Jost, J. Burkholder, T. P. Bui, W. H. Brune, S. S. Brown, C. A. Brock, I. Bourgeois, D. R. Blake, E. C. Apel, T. B. Ryerson, Proc. Natl. Acad. Sci. USA 2020, 117, 4505, https://doi.org/10.1029/2017JD02808210.1073/pnas.1919344117.

[12] B. C. McDonald, J. A. de Gouw, J. B. Gilman, S. H. Jathar, A. Akherati, C. D. Cappa, J. L. Jimenez, J. Lee-Taylor, P. L. Hayes, S. A. McKeen, Y. Y. Cui, S.-W. Kim, D. R. Gentner, G. Isaacman-VanWertz, A. H. Goldstein, R. A. Harley, G. J. Frost, J. M. Roberts, T. B. Ryerson, M. Trainer, Science 2018 359, 760, https://doi.org/10.1029/2017JD02808210.1126/science.aaq0524.

[13] M. M. Coggon, B. C. McDonald, A. Vlasenko, P. R. Veres, F. Bernard, A. R. Koss, B. Yuan, J. B. Gilman, J. Peischl, K. C. Aikin, J. DuRant, C. Warneke, S.-M. Li, J. A. de Gouw, Environ. Sci. Technol. 2018, 52, 5610, https://doi.org/10.1029/2017JD02808210.1021/acs.est.8b00506.

[14] Ten health issues WHO will tackle this year, https://www.who.int/news-room/spotlight/ten-threats-to-global-healthin-2019, accessed October 30, 2021.

[15] T. Bruderer, T. Gaisl, M. T. Gaugg, N. Nowak, B. Streckenbach, S. Müller, A. Moeller, M. Kohler, R. Zenobi, Chem. Rev. 2019, 119, 10803, https://doi.org/10.1021/acs.chemrev.9b00005.

[16] T. Bruderer, M. T. Gaugg, L. Cappellin, F. Lopez-Hilfiker, M. Hutterli, N. Perkins, R. Zenobi, A. Moeller, J. Am. Soc. Mass Spectrom. 2020, 31, 1632 https://doi.org/10.1021/jasms.0c00059.

[17] A. Fernandez-Montero, J. Argemi, J. A. Rodríguez, A. H. Ariño, L. Moreno-Galarraga, EClinicalMedicine 2021, 37, 100954 , https://doi.org/10.1016/j.eclinm.2021.100954.

[18] (VIDEO) French TV News Highlights COVID Detection Using the Vocus PTR-TOF -, https://www.tofwerk.com/french-news-covid-19-detection-vocus-ptr-tof/, accessed October 30, 2021.

[19] M. Erb, Curr. Opin. Plant Biol. 2018, 44, 117 , https://doi.org/10.1016/j.pbi.2018.03.008.

[20] Integrated Vocus PTR-TOF with Autosampler for VOC Monitoring: Video, https://www.tofwerk.com/vocus-ptr-tof-autosampler-voc-monitoring-video/, accessed October 30, 2021.

[21] H. R. Buser, C. Zanier, H. Tanner, J. Agric. Food Chem. 1982, 30, 359, https://doi.org/10.1021/jf00110a037.

[22] L. Cappellin, F. D. Lopez-Hilfiker, V. Pospisilova, L. Ciotti, P. Pastore, M. Gonin, M. A. Hutterli, Anal. Chem. 2020, 92, 9823, https://doi.org/10.1021/acs.analchem.0c01326.

[23] Vocus Cork Analyzer - Industrial solution for cork off-flavor analysis, https://www.tofwerk.com/products/vocus-cork-analyzer/, accessed October 30, 2021.

\section{License and Terms}

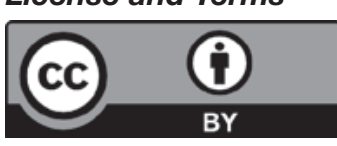

This is an Open Access article under the terms of the Creative Commons Attribution License CC BY 4.0. The material may not be used for commercial purposes.

The license is subject to the CHIMIA terms and conditions: (https://chimia.ch/chimia/about).

The definitive version of this article is the electronic one that can be found at https://doi.org/10.2533/chimia.2022.60 